\title{
FAST DETECTION AND REFINED SCALE ESTIMATION USING COMPLEX ISOTROPIC WAVELETS
}

\author{
Zsuzsanna Püspöki, John Paul Ward, Daniel Sage and Michael Unser
}

\author{
Biomedical Imaging Group, École Polytechnique Fédérale the Lausanne, Switzerland
}

\begin{abstract}
The dyadic scaling in the discrete wavelet transform can lead to a loss of precision, in comparison to the computationally unrealistic continuous wavelet transform. To overcome this obstacle, we propose a novel method to locally scale wavelets between dyadic scales in an efficient way. We compute complex wavelet coefficients for a tight frame with a dyadic scale progression. Our isotropic complex wavelets are designed such that the deviation from the nominal scale is encoded in the phase of the coefficients. Moreover, the magnitude of the coefficients is used for feature detection. Numerical experiments are presented to justify our method, and we present results for feature extraction from real data.
\end{abstract}

Index Terms - scalable filters, complex isotropic wavelets, local scale estimation, tight wavelet frames

\section{INTRODUCTION}

Wavelets are one of the most efficient and widely used processing tools for feature extraction in image analysis. They provide a unifying framework for decomposing images in a multi-scale hierarchy, and thus have many successful applications, such as contour detection, image filtering, orientation and texture analysis, synthesis and so forth (e.g., [1,2]).

One of the main advantages of wavelet methods is that they provide a high level abstraction from the data, thus one can capture or isolate information related to a particular aspect (e.g., frequency or spatial location). However, in classical wavelet schemes, the between-scale details are diffused among the wavelet coefficients and the precise size of a particular object cannot be determined easily.

The continuous wavelet transform theoretically provides a solution, but its implementation, storage and computational cost is prohibitive for image processing.

In this paper, we propose the construction of locally scalable filters based on tight wavelet frames in order to overcome their restrictive dyadic scale hierarchy and obtain between-scale information. We design discrete wavelet frames that can be transformed to approximate a continuous wavelet transform.

Theories addressing local geometrical transformations have appeared in the literature since the early 1990's. A general approach to constructing scalable functions was first given by Simoncelli et al. [3]. Perona generalized this to arbitrary compact transformations without requiring the group properties [4]. Teo, in his doctoral dissertation and a series of papers, unified the existing theories and provided a solid mathematical framework for the study of transformability based on the theory of Lie groups and Lie algebras [5].

Our approach differs from Teo's in several ways. He does not discuss wavelets, but uses his definition of local transformability to get mean-square approximations of a function. This approximation would then be exactly scalable over a limited range of parameters. We, on the other hand, are interested in discrete wavelet frames that approximate a continuous wavelet transform within a limited range of parameters in the sense of applying a localizing window to the transformation parameter.

In this paper, we design a novel scalable filter scheme based on the combination of linear filters and tight wavelet frames, described in Section 2.2, and a corresponding scale estimation algorithm that is based on a wavelet-domain phase.

\section{GENERAL FRAMEWORK}

In this paper we use $\boldsymbol{x} \in \mathbb{R}^{2}$ and $\boldsymbol{\omega} \in \mathbb{R}^{2}$ for the Cartesian, and $(r \in \mathbb{R}, \theta \in[-\pi, \pi)$ ) and $(\rho \in \mathbb{R}, \phi \in[-\pi, \pi)$ ) for the polar coordinates in spatial and Fourier domains, respectively. We denote the Fourier transform of a function $f$ by $\hat{f}$.

\subsection{Concept}

The functions

$$
[\log x]^{n} \mathrm{e}^{\alpha \log x}=[\log x]^{n} x^{\alpha}
$$

for $n \in\{0,1,2, \ldots\}$ and $\alpha \in \mathbb{C}$ are eigenfunctions of the scaling operator. Functions of the form

$$
\begin{aligned}
& {[\log x]^{n} \mathrm{e}^{a \log _{2} x} \cos \left(b \log _{2} c x\right)} \\
& {[\log x]^{n} \mathrm{e}^{a \log _{2} x} \sin \left(b \log _{2} c x\right)}
\end{aligned}
$$

with real $a, b, c$ are also covered by this result. In particular, we shall focus on the functions which are phase-factors only:

$$
\begin{aligned}
\mathrm{e}^{\mathrm{j} \omega_{0} \log _{2}(\alpha \rho)} & =\mathrm{e}^{\mathrm{j} \omega_{0} \log _{2}(\alpha)+\mathrm{j} \omega_{0} \log _{2}(\rho)} \\
& =\mathrm{e}^{\mathrm{j} \omega_{0} \log _{2}(\alpha)} \mathrm{e}^{\mathrm{j} \omega_{0} \log _{2}(\rho)} .
\end{aligned}
$$


Here the two-dimensional function is radial, with $\rho=|\omega|$. As we can see, this function can be dilated by applying a simple scalar multiplication. The dependence on $\alpha$ is cyclic: if the ratio $\alpha / \alpha^{\prime}$ is of the form $2^{2 \pi n / \omega_{0}}$, then $\alpha$ and $\alpha^{\prime}$ produce equivalent changes in local scale.

Wavelets cannot be of the form shown above, so they are not eigenfunctions of the scaling operator. Thus it is not possible to design perfectly scalable wavelets. There is an intuitive interpretation: wavelets are localized in time/space and in scale. But this kind of localization is in contradiction with arbitrary dilations (arbitrary scaling) by scalar multiplication.

However, it is possible to define wavelets that are locally scalable by combing the scaling eigenfunctions with a tight wavelet frame. The resulting wavelets are locally scalable in two senses: around the points of the grid, and within a range of scales (i.e. within a window in the Fourier domain). The first comes from having a basis or representation with shifts of the same function, the second is due to the use of a window function. We define those wavelets as locally scalable wavelets, and we propose to use them to capture the size of features in images.

\subsection{The locally scalable wavelet frames}

Due to the scaling property of the Fourier transform, scaling in time/space is equivalent to inverse scaling in Fourier, thus we can define our wavelet templates in the Fourier domain.

The basis functions of the wavelet frame are generated by suitable dilations and translations of a single mother wavelet $\xi(\boldsymbol{x})$ with a radial Fourier transform $\hat{\xi}(\boldsymbol{\omega})=\hat{h}(\rho)$. In this work, we use Meyer's isotropic mother wavelet ${ }^{1}$ [6], defined by its Fourier transform:

$$
\hat{h}(\rho)=\left\{\begin{array}{cc}
\sin \left(\frac{\pi}{2} v\left(\frac{4 \rho}{\pi}-1\right)\right), & \frac{\pi}{4}<\rho \leq \frac{\pi}{2} \\
\cos \left(\frac{\pi}{2} v\left(\frac{2 \rho}{\pi}-1\right)\right), & \frac{\pi}{2}<\rho \leq \pi \\
0, & \text { otherwise }
\end{array}\right.
$$

The template for our "locally scalable" tight wavelet frame is given by

$$
\begin{aligned}
\psi(x) & =\mathscr{F}^{-1}\left\{\hat{h}(\rho) \cos \left(\omega_{0} \log _{2}(\kappa \rho)\right)\right\} \\
& +\mathrm{j} \mathscr{F}^{-1}\left\{\hat{h}(\rho) \sin \left(\omega_{0} \log _{2}(\kappa \rho)\right)\right\} \\
& =\mathscr{F}^{-1}\left\{\hat{h}(\rho) \mathrm{e}^{\mathrm{j} \omega_{0} \log _{2}(\kappa \rho)}\right\} .
\end{aligned}
$$

The above template has two parameters: $\omega_{0}$ and $\kappa$ that can be tuned. In our experiments we fix these parameters to $\kappa=2^{5} / \pi$ and $\omega_{0}=4 \pi$.

\footnotetext{
${ }^{1}$ For the Meyer wavelet of order $N$, the auxiliary function $v(t)$ is a polynomial of degree $2 N+1$, which is chosen such that: $v(t)=0$, if $t \leq 0$, $v(t)=1$, if $t \geq 1$, and $v(t)+v(1-t)=1$, while $v \in C^{N}([0,1])$. E.g., the auxiliary function that achieves a frequency response with $N=3$ continuous derivatives is $v(t)=t^{4}\left(35-84 t+70 t^{2}-20 t^{3}\right)$.
}

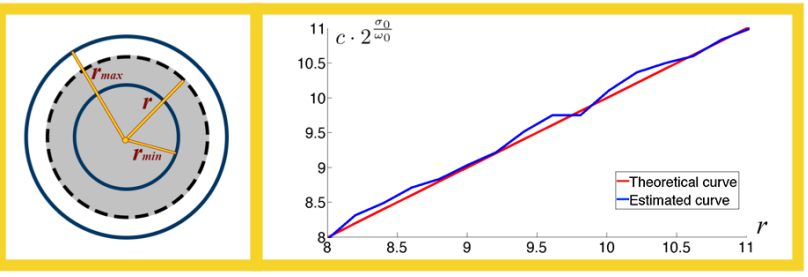

Fig. 1. Reference images (left) and size measurements (right). The graph shows the measured blob size (radius extracted from the phase of the wavelet coefficients, blue) as a function of the actual blob size (radius). The red curve represents the theoretical values in the continuous case. $c$ is a normalization constant.

Proposition 1. Let $\left\{\xi_{i, \boldsymbol{k}}\right\}$ be a radial wavelet tight frame of $L_{2}\left(\mathbb{R}^{2}\right)$ generated from the mother wavelet $\xi=\xi_{0,0}$ by defining

$$
\xi_{i, \boldsymbol{k}}(\boldsymbol{x})=2^{-i} \xi\left(\frac{\boldsymbol{x}}{2^{i}}-\boldsymbol{k}\right),
$$

where $\xi$ fulfills the conditions of Proposition 4.1 in [7], with the additional assumption that $\hat{\xi}(\omega)=0$ for $\omega$ in the neighborhood of $\mathbf{0}$. Then,

$$
\psi=\mathscr{F}^{-1}\left\{\mathrm{e}^{\mathrm{j} \omega_{0} \log _{2}(\kappa \rho)} \hat{\xi}(\omega)\right\}
$$

defines a locally scalable tight wavelet frame by shifts and dilations. The new frame functions are related to the original wavelets by

$$
\psi_{i, \boldsymbol{k}}=\mathscr{F}^{-1}\left\{\mathrm{e}^{\mathrm{j} \omega_{0} \log _{2}\left(2^{i} \kappa \rho\right)} \hat{\xi}_{i, \boldsymbol{k}}\right\} .
$$

We can interpret the local dilation of $\psi$ by a factor $\alpha$ in the frequency band defined by $\hat{h}(\rho)$ and centered at a given point. In the Fourier domain,

$$
\begin{aligned}
\hat{h}(\rho) \mathrm{e}^{\mathrm{j} \omega_{0} \log _{2}(\alpha \kappa \rho)} & =\mathrm{e}^{\mathrm{j} \omega_{0} \log _{2} \alpha} \hat{h}(\rho) \mathrm{e}^{\mathrm{j} \omega_{0} \log _{2}(\kappa \rho)} \\
& =\mathrm{e}^{\mathrm{j} \omega_{0} \log _{2} \alpha} \hat{\psi}(\rho) .
\end{aligned}
$$

This shows that a local dilation of $\psi$ by $\alpha$ can be obtained by multiplying $\psi$ with $\mathrm{e}^{\mathrm{j} \omega_{0} \log _{2} \alpha}$.

\subsection{Illustration}

The primary feature of our new complex wavelets is that the scale is encoded in the phase of the wavelet coefficients. To illustrate the idea we generate a series of test images where we place a blob at the center of the images. We increase the radius of the blob from 8 pixels to 11 pixels with step size 0.2 . Figure 1 presents the reference images (left) and the correspondence between the measured blob size (extracted from the phase of the wavelet coefficients) and the actual blob size. 


\subsection{Description of the scale estimation algorithm}

We shall now describe an application of these novel wavelets for the detection and estimation of roundish objects, such as cell nuclei, in images. Our blob detection and scale estimation algorithm has the following main steps.

(1) (Wavelet analysis)

We decompose the image with our locally scalable complex wavelets presented before.

(2) (Blob detection)

The detections of locations are made by looking for the local maxima of the amplitude of the wavelet coefficients.

(3) (Radius computation)

In view of (2), the local scale of a function $f$ at each location and for each wavelet bandwidth can be deduced from the phase of its analysis coefficients corresponding to locally scalable wavelets at any given point. Specifically, it is given by $2^{\sigma_{0} / \omega_{0}}$, where $\sigma_{0}$ is the phase of the wavelet coefficient at the location and scale of interest.

\section{EXPERIMENTAL RESULTS}

To evaluate the performance of the algorithm, we tested it on a variety of synthetic images in the presence of noise, and finally on actual microscope images. The aim of this section is to reveal the following properties of the method: translation invariance, robustness against noise, correct scale identification. We compare our methods with two popular blob detection tools: Difference of Gaussians (DoG), and the Hough transform for circles. In case of the DoG method we apply local maxima detection over a wide range of Gaussian smoothing. Our interest is to (i.) evaluate our method, and show that it provides a precise detection of image structures even in presence of noise; and (ii.) compare the different methods with each other.

We generate a series of test images (of size $512 \times 512$ ) with 100 randomly placed blobs of various randomly generated radii between 6 and 12 pixels. We do allow overlap between the blobs. In [8], Sage et al. showed experimentally that the spectral power density of fluorescent microscopic images is isotropic and corresponds to a fractional Brownian motion model. When generating our test images, we represent the background signal (autofluorescence) such way. We corrupt the images with isotropic Brownian motion, with a mean of zero and standard deviation of 0 to 1 . The goal is to determine the location and the size of the blobs.

To fairly compare the different methods with each other, we count the strongest 100 detections they provide. To make a quantitative evaluation we compute the Jaccard index (size of the intersection divided by the size of the union of the reference and detection sets), and in case of matched detections, the position and radius root mean square errors (RMSE in pixels).

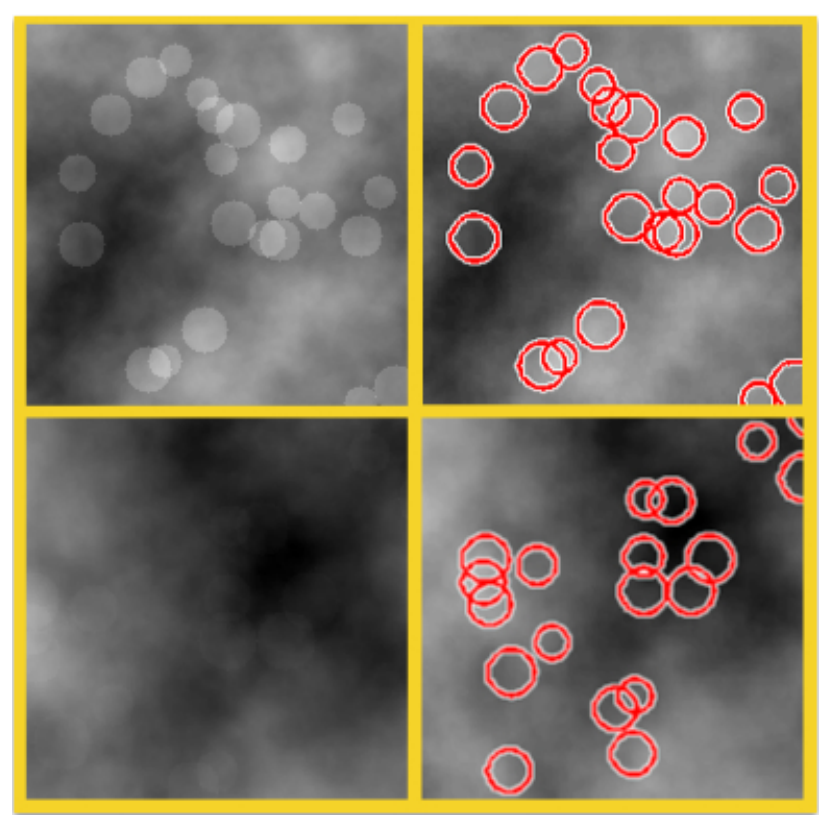

Fig. 2. Reference images (left) and detections (right). All detections are correct. Noise level: $\sigma=0.1$ and $\sigma=0.5$.

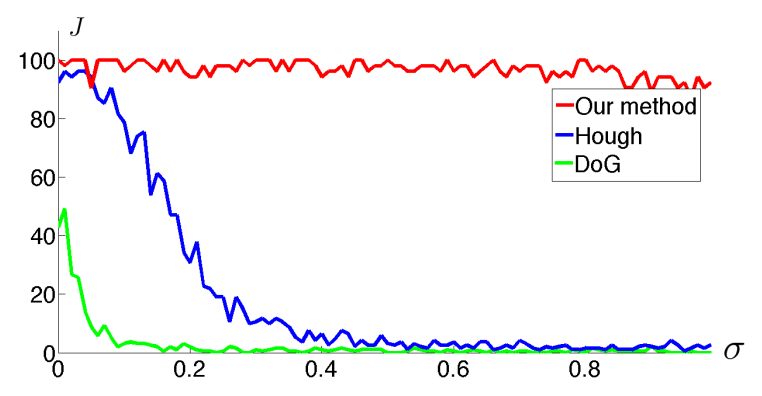

Fig. 3. Jaccard index under isotropic Brownian motion, as a function of the standard deviation of the noise $(\sigma)$.

An illustration of the results (crop of two of the test images) can be seen in Figure 2. The Jaccard index is presented in Figure 3 and the RMS errors in Figure 4.

Based on Figure 3, we can say that DoG and Hough methods perform poorly in noisy images. In particular, when the noise level is higher than a certain threshold (0.1 for the DoG, and 0.4 for the Hough), there are almost no good detections. However, with our method we can keep a high performance within a wide range of noise strengths. As mentioned before, we compute the RMS errors only in case of good detections. It implies that there is no need to make such computations for the DoG and Hough methods after the Jaccard index drops significantly.

Based on Figure 4, we can say that (i.) our method has better precision than the other methods, (ii.) the radius estimation performance of the algorithm is high under a wide range of noise powers. 

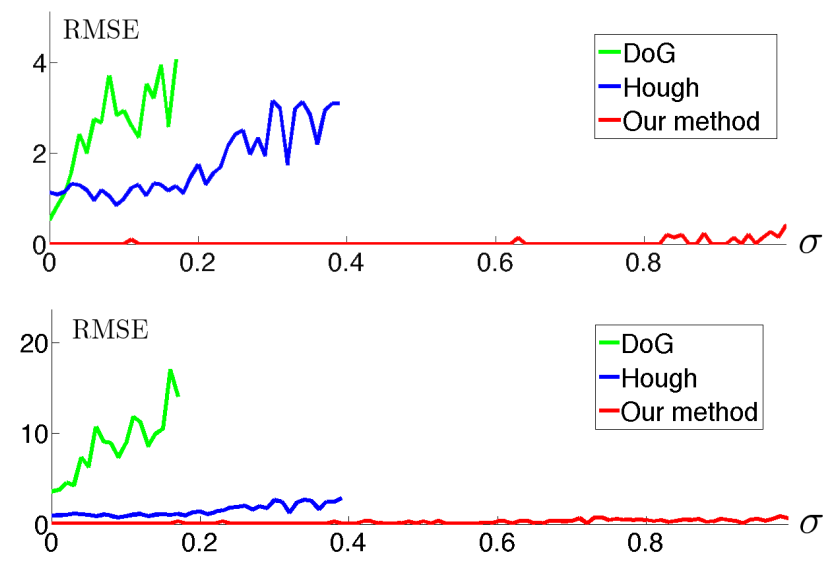

Fig. 4. Position and radius approximation error in the sense of RMSE under isotropic Brownian motion, as a function of the standard deviation of the noise $(\sigma)$.

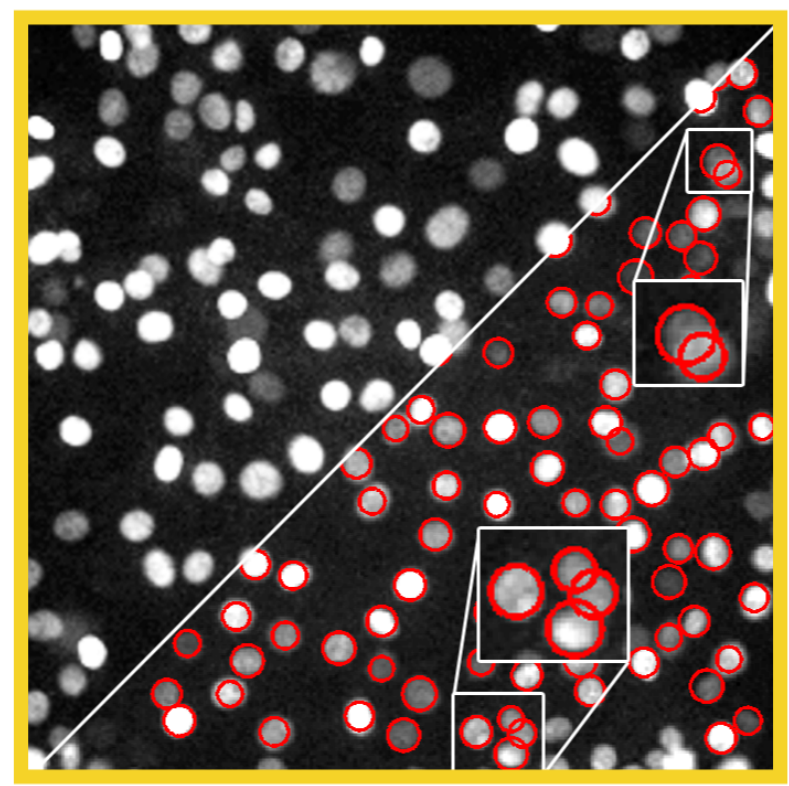

Fig. 5. Snapshot of moving cell nuclei (Courtesy of C. Dibner, University of Geneva). The results are overlaid in red in the lower half.

Figure 5 is a snapshot of moving cell nuclei. In this case, the goal was to estimate the size of different nuclei at given locations. Our method again yields good results, which suggests that our algorithm has direct applicability to bio-image analysis. The execution time of our method is a fraction of a second in an image of size $512 \times 512$.

\section{CONCLUSION}

We proposed a novel kind of complex wavelet transform to extract fine variations in scale that might be lost with clas- sical dyadic scale wavelet schemes. This framework can be used to determine the accurate size of structures in biological images. The construction of our wavelet frames makes it possible to locally scale them in a continuous range of scales by simple complex multiplication, and also to infer the local scale of objects in the image from the phase of complex wavelets coefficients. We presented practical evaluation of our approach in detecting the size of objects in images, using synthetic and real-world examples. The determination of the blob size is very effective and fast.

\section{ACKNOWLEDGMENT}

The research leading to these results has received funding from the European Research Council under the European Union's Seventh Framework Programme (FP7/2007-2013) / ERC grant agreement ${ }^{\circ} 267439$. This work was partially supported by the Hasler Foundation and the Indo-Swiss Joint Research Program.

\section{REFERENCES}

[1] C. Damerval and S. Meignen, "Blob detection with wavelet maxima lines," IEEE Signal Processing Letters, vol. 14, no. 1, pp. 39-42, Jan 2007.

[2] N. Chenouard and M. Unser, "3D steerable wavelets in practice," IEEE Transactions on Image Processing, vol. 21, no. 11, pp. 4522-4533, November 2012.

[3] E.P. Simoncelli, W.T. Freeman, E.H. Adelson, and D.J. Heeger, "Shiftable multiscale transforms," IEEE Transactions on Information Theory, vol. 38, no. 2, pp. 587607, Mar. 1992.

[4] P. Perona, "Deformable kernels for early vision," IEEE Transactions Pattern Analysis and Machine Intelligence, vol. 17, no. 5, pp. 488-499, May 1995.

[5] P.C. Teo, Theory and Applications of Steerable Functions, Ph.D. thesis, Stan. Univ., Stan., CA, USA, 1998.

[6] I. Daubechies, Ten Lectures on Wavelets, SIAM, Phil., PA, USA, 1992.

[7] M. Unser and N. Chenouard, "A unifying parametric framework for 2D steerable wavelet transforms," SIAM Journal on Imaging Sciences, vol. 6, no. 1, pp. 102-135, 2013.

[8] D. Sage, F.R. Neumann, F. Hediger, S.M. Gasser, and M. Unser, "Automatic tracking of individual fluorescence particles: application to the study of chromosome dynamics," IEEE Transactions on Image Processing, vol. 14, no. 9, pp. 1372-1383, Sept 2005. 